\title{
Gas of wormholes in Euclidean quantum field theory
}

\author{
E.P. Savelova* \\ Dubna International University of Nature, Society and Man, \\ Universitetskaya Str. 19, Dubna, 141980, Russia
}

(Dated:)

\begin{abstract}
We model the spacetime foam picture by a gas of wormholes in Euclidean field theory. It is shown that at large distances the presence of such a gas leads merely to a renormalization of mass and charge values. We also demonstrate that there exist a class of specific distributions of point-like wormholes which essentially change the ultraviolet behavior of Green functions and lead to finite quantum field theories.
\end{abstract}

PACS numbers: 04.60.-m, 04.60.Bc, 03.70.+k

\section{INTRODUCTION}

It is widely expected that at very small scales spacetime has a foam-like structure [1] and from the very beginning it was expected that the spacetime foam picture may solve the problem of divergencies in quantum field theory. The most rigorous description of the spacetime foam can be achieved in the approach which uses the lattice quantum gravity e.g., see [2, 3] and references therein. In particular, it allows to calculate the spectral dimension for nonperturbative quantum gravity [4] which seems to run from a value of $D=3 / 2$ at short distance to $D=4$ at large distance scales. However, such an approach requires numerical investigations which are not sufficiently visual. One would like to have a picture in which basic properties of the spacetime foam become more transparent and may allow to control numerical investigations.

We point out that the spacetime foam structure represents quantum topology fluctuations and roughly it can be described by the picture when at very small scales space is filled with a gas of virtual wormholes. Unlike actual wormholes virtual wormhole exists only for a very short period of time and at very small scales, and does not obey to the Einstein equations and, therefore, there are no theorems which may forbid origin of such objects. From the mathematical standpoint the simplest virtual wormhole can be described by a standard four-dimensional wormhole whose throat section is a 3 -dimensional sphere $S^{3}$ and due to its high symmetry such an object admits the rigorous mathematical treatment (analogous to [5]). In the present paper we model the spacetime foam by a gas of wormholes in Euclidean field theory and investigate the simplest spacetime foam effects.

We point out that the interest to Euclidean wormholes is not new. There was a wide investigation of Euclidean wormholes or baby universes in connection to topology changes and the loss of quantum coherence [6, 7], see also [8]. The results of observations of neutrino oscillations and other oscillations [16] $\left(\nu_{\tau} \leftrightarrow \nu_{\mu}, K \leftrightarrow K\right.$, etc. $)$ [9] suggested that there are severe constraints on the magnitude of processes of the actual changing the topology as it was predicted by [7] and that such processes at the present time are strongly suppressed. Those however do not forbid the origin of wormhole-like structures as discussed by [10]. In turn, this also means that changes in the topology in the modern Universe can be only virtual (i.e., the space-time foam).

\section{THE STRUCTURE OF A WORMHOLE}

In the Euclidean field theory the simplest wormhole can be constructed merely by a gluing procedure [11] and is described by the metric $(\alpha=1,2,3,4)$

$$
d s^{2}=h^{2}(r) \delta_{\alpha \beta} d x^{\alpha} d x^{\beta}
$$

where $h(r)=1+\theta(a-r)\left(\frac{a^{2}}{r^{2}}-1\right)$ and $\theta(x)$ is the step function. Such a wormhole has vanishing throat length. Indeed, in the region $r>a, h=1$ and the metric is flat, while the region $r<a$, with the obvious transformation $y^{\alpha}=\frac{a^{2}}{r^{2}} x^{\alpha}$, is also flat for $y>a$. Therefore, the regions $r>a$ and $r<a$ represent two Euclidean spaces glued at the 
surface of a sphere $S^{3}$ with the centre at the origin $r=0$ and the radius $r=a$. Such a space can be described with the ordinary double-valued flat metric in the region $r_{ \pm}>a$ by

$$
d s^{2}=\delta_{\alpha \beta} d x_{ \pm}^{\alpha} d x_{ \pm}^{\beta}
$$

where the coordinates $x_{ \pm}^{\alpha}$ describe two different sheets of space. Now, identifying the inner and outer regions of the sphere $S^{3}$ allows the construction of a wormhole which connects regions in the same space (instead of two independent spaces). This is achieved by gluing the two spaces in (2) by motions of the Euclidean space. If $R_{ \pm}$is the position of the sphere in coordinates $x_{ \pm}^{\mu}$, then the gluing is the rule

$$
x_{+}^{\mu}=R_{+}^{\mu}+\Lambda_{\nu}^{\mu}\left(x_{-}^{\nu}-R_{-}^{\nu}\right)
$$

where $\Lambda_{\nu}^{\mu} \in O(4)$, which represents the composition of a translation and a rotation of the Euclidean space (Lorentz transformation). In terms of common coordinates such a wormhole represents the standard flat space in which the two spheres $S_{ \pm}^{3}$ (with centers at positions $R_{ \pm}$) are glued by the rule (3). We point out that the physical region is the outer region of the two spheres. Thus, in general, the wormhole is described by a set of parameters: the throat radius $a$, positions of throats $R_{ \pm}$, and the rotation matrix $\Lambda_{\nu}^{\mu} \in O(4)$.

\section{THE GREEN FUNCTION FOR A SINGLE WORMHOLE CONNECTING TWO SPACES}

In the Euclidean field theory the Green function obeys the Laplace equation

$$
\left(-\Delta+\zeta R+m^{2}\right) G\left(x, x^{\prime}\right)=4 \pi^{2} \delta\left(x-x^{\prime}\right)
$$

where $m$ is the mass, $R$ is the scalar curvature, $\Delta=\frac{1}{\sqrt{g}} \partial_{\alpha}\left(\sqrt{g} g^{\alpha \beta} \partial_{\beta}\right)$, and $g_{\alpha \beta}$ is the metric. In the Euclidean space the metric is flat $g_{\alpha \beta}=\delta_{\alpha \beta}$ and the above equation has the well-known solution $G_{0}\left(x, x^{\prime}\right)=m^{2} \frac{K_{1}(m r)}{m r}$, where $r^{2}=\left(x-x^{\prime}\right)^{2}$ and $K_{1}(x)$ is the modified Bessel function. When considering a space with a wormhole the metric cannot be chosen everywhere flat and the exact form of the Green function depends on the specific structure of the wormhole. In the present section we construct the Green function for the simplest wormhole connecting two Euclidean spaces which is described by the metric (11). In this case the equation (4) admits the exact solution.

Indeed, consider four-dimensional spherical coordinates $r, \chi, \theta, \phi$ connected to the cartesian coordinates by

$$
\begin{aligned}
& x=r \sin \chi \sin \theta \cos \phi, y=r \sin \chi \sin \theta \sin \phi \\
& z=r \sin \chi \cos \theta, t=r \cos \chi .
\end{aligned}
$$

where $0 \leq r<\infty, 0 \leq \phi<2 \pi$, and $0 \leq \chi, \theta<\pi$. The square of the element of length in this coordinates is

$$
d s^{2}=h^{2}(r)\left(d r^{2}+r^{2}\left[d \chi^{2}+\sin ^{2} \chi\left(d \theta^{2}+\sin ^{2} \theta d \phi^{2}\right)\right]\right) .
$$

For the Laplacian operator in spherical coordinates in a four-dimensional Euclidean space applied to a scalar $f$ we get

$$
\Delta f=\frac{1}{r^{3} h^{4}} \frac{\partial}{\partial r}\left(r^{3} h^{2} \frac{\partial f}{\partial r}\right)+\frac{1}{r^{2} h^{2}} \Delta_{\Omega} f
$$

where $\Delta_{\Omega} f=f_{; \alpha \alpha}^{; \alpha}$ is the angular part of $\Delta(\Omega=(\chi, \theta, \phi))$. The angular part of $f$ can be written in terms of four -dimensional spherical harmonics $f=\sum f_{n l m} Q_{n l m}$ e.g., see Ref. [12] where

$$
\Delta_{\Omega} Q_{n l m}=-\left(n^{2}-1\right) Q_{n l m}
$$

$(n=1,2, \ldots)$ which can be decomposed as $Q_{n l m}(\Omega)=q_{l m}^{(n)} Y_{l m}(\theta, \phi) \Pi_{n l}(\chi)$, where $q_{l m}^{(n)}$ are normalization constants, $Y_{l m}(\theta, \phi)$ are the usual three-dimensional spherical harmonics, and

$$
\Pi_{n l}(\chi)=\sin ^{l} \chi \frac{d^{l+1}(\cos n \chi)}{d(\cos \chi)^{l+1}},(l=0,1, \ldots, n-1) .
$$

(In particular, the most symmetric scalar spherical harmonic corresponds to $l=0$ and has the form $Q=\frac{\sin n \chi}{\sin \chi}$, $n=1,2,3, \ldots)$. These functions obey the normalization conditions

$$
\int_{0}^{\pi} \sin ^{2} \chi d \chi \int_{0}^{\pi} \sin \theta d \theta \int_{0}^{2 \pi} d \phi Q_{n l m}^{*}(\Omega) Q_{n^{\prime} l^{\prime} m^{\prime}}(\Omega)=\delta_{n n^{\prime}} \delta_{l l^{\prime}} \delta_{m m^{\prime}}
$$


and the completeness condition

$$
\sum_{n=1}^{\infty} \sum_{l=0}^{n-1} \sum_{m=-l}^{l} Q_{n l m}^{*}(\Omega) Q_{n l m}\left(\Omega^{\prime}\right)=\frac{1}{\sin ^{2} \chi \sin \theta} \delta\left(\Omega-\Omega^{\prime}\right) .
$$

The Euclidean Green function (for $m=0$ case) has the decomposition

$$
G_{0}\left(x, x^{\prime}\right)=\frac{1}{\left(x-x^{\prime}\right)^{2}}=\sum_{n=1}^{\infty} \frac{x_{<}^{n-1}}{x_{>}^{n+1}} Q_{n}(\widetilde{\chi}) .
$$

where $x_{>}$and $x_{<}$are the biggest and smallest value of $x, x^{\prime}$ respectively and we have used the composition theorem for spherical harmonics

$$
Q_{n}(\widetilde{\chi})=\frac{4 \pi^{2}}{2 n} \sum_{l=0}^{n-1} \sum_{m=-l}^{l} Q_{n l m}^{*}\left(\Omega^{\prime}\right) Q_{n l m}(\Omega)
$$

where $\tilde{\chi}$ is the angle between the vectors $x$ and $x^{\prime}$.

Consider the decomposition of $\delta$-function as

$$
\delta\left(x-x^{\prime}\right)=\frac{1}{r^{3}} \delta\left(r-r^{\prime}\right) \sum_{n=1}^{\infty} \sum_{l=0}^{n-1} \sum_{m=-l}^{l} Q_{n l m}^{*}(\Omega) Q_{n l m}\left(\Omega^{\prime}\right) .
$$

Then we may represent the Green function in the same form

$$
G\left(x, x^{\prime}\right)=\sum_{n=1}^{\infty} \sum_{l=0}^{n-1} \sum_{m=-l}^{l} Q_{n l m}^{*}\left(\Omega^{\prime}\right) g_{n}\left(r, r^{\prime}\right) Q_{n l m}(\Omega)
$$

which gives from (4)

$$
-\frac{1}{r^{3} h^{4}} \frac{\partial}{\partial r}\left(r^{3} h^{2} \frac{\partial g_{n}}{\partial r}\right)+\left(\zeta R+m^{2}\right) g_{n}+\frac{\left(n^{2}-1\right)}{r^{2} h^{2}} g_{n}=\frac{4 \pi^{2}}{r^{3} h^{4}} \delta\left(r-r^{\prime}\right) .
$$

Here the scalar curvature with the metric (1) is [11] (the step function at the junction requires considering distributional curvature etc., see details in [13])

$$
R=-\frac{12}{a} \delta(r-a) .
$$

Consider the region $r>a$ which corresponds to the first sheet of the Euclidean space. Then $h=1$ and if $r \neq r^{\prime}$ the above equation gives

$$
\frac{\partial^{2} g_{n}}{\partial x^{2}}+\frac{3}{x} \frac{\partial g_{n}}{\partial x}-\left(1+\frac{\left(n^{2}-1\right)}{x^{2}}\right) g_{n}=0
$$

where $x=m r$. The substitution $g=f / x$ transforms it into the modified Bessel equation

$$
\frac{\partial^{2} f}{\partial x^{2}}+\frac{1}{x} \frac{\partial f}{\partial x}-\left(1+\frac{n^{2}}{x^{2}}\right) f=0
$$

whose solutions are the modified Bessel functions $I_{n}(x)$ and $K_{n}(x)$ and therefore we find

$$
g_{n}=C_{1} \frac{I_{n}(m r)}{m r}+C_{2} \frac{K_{n}(m r)}{m r} .
$$

In the region $r<a$ we use the coordinate transformation $y^{\alpha}=\frac{a^{2}}{r^{2}} x^{\alpha}$ which does not change the angular dependence, while it transforms $r \rightarrow \widetilde{r}=a^{2} / r$. Thus the region $r<a$ corresponds to the second sheet of the Euclidean space $\widetilde{r}>a$ where we get the same solution (8), or

$$
g_{n}=\widetilde{C}_{1} \frac{I_{n}\left(m a^{2} / r\right)}{m a^{2} / r}+\widetilde{C}_{2} \frac{K_{n}\left(m a^{2} / r\right)}{m a^{2} / r}
$$


We recall that these functions has the well-known asymptotic as $x \rightarrow 0$

$$
\frac{I_{n}(x)}{x} \rightarrow \frac{1}{2 \Gamma(n+1)}\left(\frac{x}{2}\right)^{n-1}, \frac{K_{n}(x)}{x} \rightarrow \frac{\Gamma(n)}{4}\left(\frac{2}{x}\right)^{n+1}
$$

and as $x \rightarrow \infty$

$$
I_{n}(x) \rightarrow e^{x}\left(\frac{1}{\sqrt{2 \pi x}}+o\left(x^{-3 / 2}\right)\right), K_{n}(x) \rightarrow e^{-x} \sqrt{\frac{\pi}{2 x}}\left(1+o\left(\frac{1}{x}\right)\right) .
$$

Now to get the solution to (77) we have to consider the three regions $0<r<a, a<r<r^{\prime}$, and $r>r^{\prime}$ where the solution has the form (9) and (8) respectively, and match this functions by the boundary conditions. At the boundary $r=a$ and at the source $r=r^{\prime}$ we get

$$
\begin{gathered}
\left.\frac{\partial g_{n}}{\partial r}\right|_{a-0} ^{a+0}=-\zeta \frac{12}{a} h^{2} g_{n}(a) \\
\left.\frac{\partial g_{n}}{\partial r}\right|_{r^{\prime}-0} ^{r^{\prime}+0}=-\frac{4 \pi^{2}}{r^{\prime 3} h^{2}} \\
g_{n}(a-0)=g_{n}(a+0) \\
g_{n}\left(r^{\prime}-0\right)=g_{n}\left(r^{\prime}+0\right),
\end{gathered}
$$

while the two more boundary conditions we get from the requirement $g \rightarrow 0$ as $r, \widetilde{r} \rightarrow \infty$, which give as $r<a$

$$
g_{n}=\widetilde{C} \frac{K_{n}\left(m \frac{a^{2}}{r}\right)}{m \frac{a^{2}}{r}}
$$

and as $r>r^{\prime}$

$$
g_{n}=C \frac{K_{n}\left(m r^{\prime}\right)}{m r^{\prime}}
$$

The jump of derivatives at positions $r=r^{\prime}$ and $r=a$ means the presence of sources. At $r=r^{\prime}$ we have the standard external source from the right hand side of (7), while at $r=a$ the source generates due to the curvature. The explicit form of the function $g_{n}$ in every of regions is

$$
g_{n}= \begin{cases}A \frac{K_{n}\left(m \frac{a^{2}}{r}\right)}{m \frac{a^{2}}{r}} & \text { as } 0<r<a ; \\ B \frac{I_{n}(m r)}{m r}+C \frac{K_{n}(m r)}{m r}, & \text { as } a<r<r^{\prime} \\ D \frac{K_{n}(m r)}{m r}, & \text { as } r^{\prime}<r<\infty ;\end{cases}
$$

where the coefficients are expressed as

$$
\begin{gathered}
B=4 \pi^{2} m^{2} \frac{K_{n}\left(m r^{\prime}\right)}{m r^{\prime}} \\
C=-\frac{\left(\ln \left(\frac{I_{n}(m a)}{m a} \frac{K_{n}(m a)}{m a}\right)\right)^{\prime}+\frac{12}{a} \zeta}{2\left(\left(\ln \frac{K_{n}(m a)}{m a}\right)^{\prime}+\frac{6}{a} \zeta\right)} \frac{\frac{I_{n}(m a)}{m a}}{\frac{K_{n}(m a)}{m a}} B \\
D=C+4 \pi^{2} m^{2} \frac{I_{n}\left(m r^{\prime}\right)}{m r^{\prime}}
\end{gathered}
$$




$$
A=-C-\frac{\left(\ln \frac{I_{n}(m a)}{m a}\right)^{\prime}+\frac{6}{a} \zeta}{\left(\ln \frac{K_{n}(m a)}{m a}\right)^{\prime}+\frac{6}{a} \zeta} \frac{\frac{I_{n}(m a)}{m a}}{\frac{K_{n}(m a)}{m a}} B
$$

where $\left(\frac{K_{n}(m a)}{m a}\right)^{\prime}=\frac{\partial}{\partial a} \frac{K_{n}(m a)}{m a}$. Thus we find for the Green function the expressions as $r<a$

$$
g_{n}=\left(\beta_{n}\left(a, r^{\prime}\right)-\alpha_{n}\left(a, r^{\prime}\right)\right) g_{n}^{0}(\widetilde{r}, a)
$$

and as $r>a$

$$
g_{n}=g_{n}^{0}\left(r, r^{\prime}\right)+\alpha_{n}\left(a, r^{\prime}\right) g_{n}^{0}(r, a)
$$

where

$$
\begin{gathered}
\alpha_{n}\left(a, r^{\prime}\right)=-\frac{\left(\ln \left(\frac{I_{n}(m a)}{m a} \frac{K_{n}(m a)}{m a}\right)\right)^{\prime}+\frac{12}{a} \zeta}{2\left(\left(\ln \frac{K_{n}(m a)}{m a}\right)^{\prime}+\frac{6}{a} \zeta\right)} \frac{\frac{K_{n}\left(m r^{\prime}\right)}{m r^{\prime}}}{\frac{K_{n}(m a)}{m a}}, \\
\beta_{n}\left(a, r^{\prime}\right)=-\frac{\left(\ln \frac{I_{n}(m a)}{m a}\right)^{\prime}+\frac{6}{a} \zeta}{\left(\ln \frac{K_{n}(m a)}{m a}\right)^{\prime}+\frac{6}{a} \zeta} \frac{\frac{K_{n}\left(m r^{\prime}\right)}{m r^{\prime}}}{\frac{K_{n}(m a)}{m a}},
\end{gathered}
$$

and $g_{n}^{0}$ is the standard Euclidean Green function which is given by

$$
g_{n}^{0}\left(r, r^{\prime}\right)=4 \pi^{2} m^{2} \frac{K_{n}\left(m r_{>}\right)}{m r_{>}} \frac{I_{n}\left(m r_{<}\right)}{m r_{<}}
$$

where $r_{>}$and $r_{<}$denote the biggest and smallest value of $r$ and $r^{\prime}$ respectively.

In the massless case $m=0$ we find [11] as $r<a$

$$
g_{n}=\left(\frac{6 \zeta-1}{(6 \zeta-(n+1))}-\frac{6 \zeta+n-1}{6 \zeta-(n+1)}\right)\left(\frac{a}{r^{\prime}}\right)^{n+1} g_{n}^{0}(\widetilde{r}, a)
$$

and as $r>a$

$$
g_{n}^{0}+\Delta g_{n}=g_{n}^{0}\left(r, r^{\prime}\right)-\frac{6 \zeta-1}{(6 \zeta-(n+1))}\left(\frac{a}{r^{\prime}}\right)^{n+1} g_{n}^{0}(r, a)
$$

where $g_{n}^{0}$ is given by (12). Or explicit expressions as $r<a$

$$
g_{n}=\frac{4 \pi^{2}}{2 n} \frac{n}{n+1-6 \zeta}\left(\frac{a^{2}}{r^{\prime} \widetilde{r}}\right)^{n-1}\left(\frac{a}{r^{\prime} \widetilde{r}}\right)^{2}
$$

and as $r>a$

$$
g_{n}^{0}+\Delta g_{n}=\frac{4 \pi^{2}}{2 n}\left(\frac{1}{r_{>}}\right)^{2}\left(\frac{r_{<}}{r_{>}}\right)^{n-1}-\frac{4 \pi^{2}}{2 n} \frac{1-6 \zeta}{n+1-6 \zeta}\left(\frac{a^{2}}{r r^{\prime}}\right)^{n-1}\left(\frac{a}{r^{\prime} r}\right)^{2} .
$$

In particular, conformal coupling corresponds to $\zeta=1 / 6$ and in the region $r>a$ we get $\Delta g_{n}=0$ as it should be. In this case the Green function can be constructed by the image method (e.g., in three dimensions such an approach was used in [5]) or by conformal transformation of the standard Euclidean Green function. In the case of minimal coupling $\zeta=0$ we get the expressions presented in [11].

In what follows we shall compare this solution with the case when the wormhole is absent. Then the total space consists of a couple of sheets of the standard Euclidean spaces $R_{+}^{4}$ and $R_{-}^{4}$ and the total Green function consists also of the two parts $G_{0}^{+}$and $G_{0}^{-}$. Let the source belong to the first space $x^{\prime} \in R_{+}^{4}$. Then we get $G_{0}^{-}=0$, while $G_{0}^{+}$ corresponds to the standard Euclidean Green function $G_{0}$, i.e. (12). In the presence of the wormhole it is convenient to extract the part related to the standard Green function, i.e., to define the difference $G=G_{0}+\Delta G$. Then in the region $r>a$ (i.e., as $x=x_{+} \in R_{+}^{4}$ ) and in the region $r<a$ (i.e., $\widetilde{r}>a$, or $x=x_{-} \in R_{-}^{4}$ ) we get the expression

$$
\Delta g_{n}^{ \pm}=\frac{1}{2}\left(\beta_{n}\left(a, r^{\prime}\right) \pm\left(2 \alpha_{n}\left(a, r^{\prime}\right)-\beta_{n}\left(a, r^{\prime}\right)\right)\right) g_{n}^{0}(r, a) .
$$




\section{THE BIAS OF THE SOURCE, PARTIAL AMPLITUDES}

In this section we find the charge density generated on the surface of the wormhole throat. To this end we consider the equivalent problem. Indeed the true Green function obeys the equation (we recall that in the Euclidean space $R=0)$

$$
-\left(\Delta-m^{2}\right) G_{+}\left(x, x^{\prime}\right)=4 \pi^{2}\left(\delta\left(x-x^{\prime}\right)+b_{+}\left(x, x^{\prime}\right)\right)
$$

as $x, x^{\prime} \in R_{+}^{4}$ and

$$
-\left(\Delta-m^{2}\right) G_{-}\left(x, x^{\prime}\right)=4 \pi^{2} b_{-}\left(x, x^{\prime}\right)
$$

as $x \in R_{-}^{4}$. The physical region corresponds to $r_{ \pm} \geq a$ in both spaces. Thus we find

$$
G_{ \pm}\left(x, x^{\prime}\right)=G_{0}^{ \pm}\left(x, x^{\prime}\right)+\Delta G_{ \pm}\left(x, x^{\prime}\right)
$$

where $G_{0}^{-}=0$ since $x^{\prime} \in R_{+}^{4}$ and $G_{0}^{+}=G_{0}$ is the standard Euclidean Green function. The additional sources are generated on the surface of the sphere $r=a$ and therefore

$$
b_{ \pm}\left(x, x^{\prime}\right)=\sigma_{ \pm}\left(x, x^{\prime}\right) \frac{1}{a^{3}} \delta(r-a) .
$$

Consider the decomposition

$$
\sigma_{ \pm}\left(x, x^{\prime}\right)=\sum \sigma_{n l m}^{ \pm}\left(a, x^{\prime}\right) Q_{n l m}(\Omega)
$$

where $\Omega$ is the angular part of the coordinates $x$. Then we find

$$
\Delta G_{ \pm}\left(x, x^{\prime}\right)=\int G_{0}\left(x, x^{\prime \prime}\right) \sigma_{ \pm}\left(a, x^{\prime}\right) \frac{1}{a^{3}} \delta\left(r^{\prime \prime}-a\right) d^{4} x^{\prime \prime}
$$

or substituting the decomposition (13) and

$$
G_{0}\left(x, x^{\prime}\right)=\sum Q_{n l m}^{*}\left(\Omega^{\prime}\right) g_{n}^{0}\left(r, r^{\prime}\right) Q_{n l m}(\Omega)
$$

we find

$$
\begin{aligned}
& \Delta G_{ \pm}\left(x, x^{\prime}\right)= \int \sum Q_{n l m}^{*}\left(\Omega^{\prime \prime}\right) g_{n}^{0}\left(r, r^{\prime \prime}\right) Q_{n l m}(\Omega) \times \\
& \times \sum \sigma_{n^{\prime} l^{\prime} m^{\prime}}^{ \pm}\left(a, x^{\prime}\right) Q_{n^{\prime} l^{\prime} m^{\prime}}\left(\Omega^{\prime \prime}\right) \frac{1}{a^{3}} \delta\left(r^{\prime \prime}-a\right) d^{4} x^{\prime \prime} \\
& \Delta G_{ \pm}\left(x, x^{\prime}\right)=\sum \sigma_{n l m}^{ \pm}\left(a, x^{\prime}\right) g_{n}^{0}(r, a) Q_{n l m}(\Omega)
\end{aligned}
$$

Comparing this with the solution constructed in the previous section, i.e.,

$$
\Delta G_{ \pm}\left(x, x^{\prime}\right)=\frac{1}{2} \sum\left(\beta_{n}\left(a, r^{\prime}\right) \pm\left(2 \alpha_{n}\left(a, r^{\prime}\right)-\beta_{n}\left(a, r^{\prime}\right)\right)\right) Q_{n l m}^{*}\left(\Omega^{\prime}\right) g_{n}^{0}(r, a) Q_{n l m}(\Omega)
$$

we find

$$
\sigma_{n l m}^{ \pm}\left(a, x^{\prime}\right)=\frac{1}{2}\left(\beta_{n}\left(a, r^{\prime}\right) \pm\left(2 \alpha_{n}\left(a, r^{\prime}\right)-\beta_{n}\left(a, r^{\prime}\right)\right)\right) Q_{n l m}^{*}\left(\Omega^{\prime}\right) .
$$

Thus the bias takes the form

$$
b_{ \pm}\left(x, x^{\prime}\right)=\frac{1}{a^{3}} \delta(r-a) \sum_{n=1}^{\infty} b_{n}^{ \pm}\left(a, r^{\prime}\right) Q_{n}(\widetilde{\chi})
$$

where

$$
b_{n}^{ \pm}\left(a, r^{\prime}\right)=\frac{n}{4 \pi^{2}}\left(\beta_{n}\left(a, r^{\prime}\right) \pm\left(2 \alpha_{n}\left(a, r^{\prime}\right)-\beta_{n}\left(a, r^{\prime}\right)\right)\right)
$$


and $\tilde{\chi}$ is the angle between $x$ and $x^{\prime}$ e.g., see (6). Thus, we see that the true Green function can be obtained by means of solving the standard equation in the Euclidean space with the biased source. Indeed, every source generates a set of additional sources on the inner and outer sides of the throat surface by the rule

$$
\delta\left(x-x^{\prime}\right) \rightarrow \delta\left(x-x^{\prime}\right)+b\left(x, x^{\prime}\right)
$$

where $b\left(x, x^{\prime}\right)=b_{+}\left(x, x^{\prime}\right)$ on the outer side of the throat surface (with respect to the source) and $b\left(x, x^{\prime}\right)=b_{-}\left(x, x^{\prime}\right)$ on the inner side respectively. Since coefficients $b_{n}^{ \pm}$include a small parameter $\delta_{n}=\frac{K_{n}\left(m r^{\prime}\right)}{m r^{\prime}} / \frac{K_{n}(m a)}{m a}$ which in general case $\delta_{n} \ll 1$ (e.g., in the massless case it is merely $\left(\frac{a}{r^{\prime}}\right)^{n+1}$ ), in practical calculations this allows us to restrict with smallest terms $(n=1,2)$ only.

\section{THE BIAS FOR A WORMHOLE CONNECTING REGIONS IN THE SAME SPACE}

Consider now a wormhole which connects two regions in the same Euclidean space. The rule (16) allows to construct the exact Green function in this case as well. In this case both spaces $R_{ \pm}^{4}$ are glued by the rule (3) and the true Green function obeys to the standard equation with the biased source

$$
-\left(\Delta-m^{2}\right) G\left(x, x^{\prime}\right)=4 \pi^{2}\left(\delta\left(x-x^{\prime}\right)+b_{t o t}\left(x, x^{\prime}\right)\right)
$$

where the total bias $b_{t o t}$ is constructed by the iteration procedure

$$
b_{t o t}=\sum_{s=0}^{\infty} b_{s}
$$

as follows. Let $R_{ \pm}$be the positions of the wormhole throat in the space. Then in the leading order the source $\delta\left(x-x^{\prime}\right)$ generates the bias in the form

$$
b_{0}\left(x, x^{\prime}\right)=b_{0}^{+}\left(x, x^{\prime}\right)+b_{0}^{-}\left(x, x^{\prime}\right)
$$

where

$$
b_{0}^{ \pm}\left(x, x^{\prime}\right)=\frac{1}{a^{3}} \sum_{n=1}^{\infty} \delta\left(r_{ \pm}-a\right) b_{n}^{+}\left(a, r_{ \pm}^{\prime}\right) Q_{n}\left(\widetilde{\chi}_{ \pm}\right)+\delta\left(r_{\mp}-a\right) b_{n}^{-}\left(a, r_{ \pm}^{\prime}\right) Q_{n}\left(\widetilde{\chi}_{\mp}^{\prime}\right)
$$

describes the bias generated with respect to $S_{+}^{3}$ or $S_{-}^{3}$ respectively. We also use here the obvious definitions $r_{ \pm}=$ $\left|x_{ \pm}\right|=\left|x-R_{ \pm}\right|, r_{ \pm}^{\prime}=\left|x_{ \pm}^{\prime}\right|, \cos \widetilde{\chi}_{ \pm}=\left(n_{ \pm}^{\mu} n_{ \pm \mu}^{\prime}\right)$, and $\cos \widetilde{\chi}_{ \pm}^{\prime}=\left(n_{ \pm}^{\nu}\left(\Lambda^{ \pm 1}\right)_{\nu}^{\mu} n_{\mu \mp}^{\prime}\right)(n=x / r)$. This bias takes the structure

$$
b_{0}\left(x, x^{\prime}\right)=\frac{1}{a^{3}} \rho_{0}^{+}\left(x, x^{\prime}\right) \delta\left(r_{+}-a\right)+\frac{1}{a^{3}} \rho_{0}^{-}\left(x, x^{\prime}\right) \delta\left(r_{-}-a\right)
$$

where $\rho_{0}^{ \pm}\left(x, x^{\prime}\right)=\sum b_{n}^{+}\left(a, r_{ \pm}^{\prime}\right) Q_{n}\left(\widetilde{\chi}_{ \pm}\right)+b_{n}^{-}\left(a, r_{\mp}^{\prime}\right) Q_{n}\left(\widetilde{\chi}_{ \pm}^{\prime}\right)$ are the surface densities generated on $S_{ \pm}^{3}$

In the next order every source on $S_{-}^{3}$ generates additional sources by the same scheme (18) (e.g., see [5]) and this defines the next orders bias as

$$
b_{s}\left(x, x^{\prime}\right)=b_{s}^{+}\left(x, x^{\prime}\right)+b_{s}^{-}\left(x, x^{\prime}\right)
$$

where

$$
b_{s}^{ \pm}\left(x, x^{\prime}\right)=\int b_{0}^{ \pm}\left(x, x^{\prime \prime}\right) \rho_{s-1}^{\mp}\left(x^{\prime \prime}, x^{\prime}\right) \frac{1}{a^{3}} \delta\left(r_{\mp}^{\prime \prime}-a\right) d^{4} x^{\prime \prime}
$$

and $\rho_{s}^{ \pm}$is defined by $b_{s}^{ \pm}$via the relation (19). Thus, we get the iteration scheme for constructing the total bias $b_{t o t}\left(x, x^{\prime}\right)$ and the total Green function $G_{t o t a l}\left(x, x^{\prime}\right)=G_{0}+\int G_{0}\left(x, x^{\prime \prime}\right) b_{t o t}\left(x^{\prime \prime}, x^{\prime}\right) d^{4} x^{\prime \prime}$. We point out that the decomposition of the bias (17) includes a small parameter $\delta \leq \frac{K_{1}(m R)}{m R} / \frac{K_{1}(m a)}{m a} \leq a^{2} / R^{2} \ll 1$, where $R=|X|$ and $X=R_{+}-R_{-}$is the distance between throat centers and, therefore, the iterations converge very rapidly. 


\section{THE BIAS IN A GAS OF WORMHOLES (THE RAREFIED GAS APPROXIMATION)}

The exact form of the bias for a gas of wormholes is constructed by the same scheme described in the previous section and consists of the two steps. First, one has to define the bias for a single wormhole (17) and then one may use iterations. Let $\xi=\left(a, R_{ \pm}, \Lambda\right)$ be wormhole parameters and, therefore the bias (17) is a function of $\xi$ i.e., $b_{t o t}\left(x, x^{\prime}, \xi\right)$. Then in the first approximation the bias is merely the sum

$$
b_{\text {gas }}^{0}\left(x, x^{\prime}\right)=\sum_{A} b_{t o t}\left(x, x^{\prime}, \xi^{A}\right)
$$

In the next order every first order source generates additional sources (multiple scattering of the basic signal) as

$$
b_{\text {gas }}^{1}\left(x, x^{\prime}\right)=\sum_{A \neq B} \int b_{t o t}\left(x, x^{\prime \prime}, \xi^{A}\right) b_{t o t}\left(x^{\prime \prime}, x^{\prime}, \xi^{B}\right) d^{4} x^{\prime \prime}
$$

etc. By iterating this procedure we may find the exact form of the bias for the gas of wormholes

$$
b_{\text {gas }}\left(x, x^{\prime}\right)=\sum b_{g a s}^{s}\left(x, x^{\prime}\right)
$$

In the present section we shall consider a dilute gas approximation when characteristic parameters $a^{2} / r^{2}, a^{2} / R^{2}$, and $a^{2} / R_{A B}^{2} \ll 1$. In this case it is sufficient to retain in (14) the lowest $n=1$ (monopole) term only and neglect mutual scattering between throats of wormholes (i,e, retain $s=0$ term in (17) and (21), e.g., see for details Ref. [5]). Then the bias for a single wormhole takes the form

$$
b^{0}\left(x, x^{\prime}, \xi\right)=\frac{1}{a^{3}} \delta\left(r_{+}-a\right)\left(b_{1}^{+}\left(a, r_{+}^{\prime}\right)+b_{1}^{-}\left(a, r_{-}^{\prime}\right)\right)+\frac{1}{a^{3}} \delta\left(r_{-}-a\right)\left(b_{1}^{-}\left(a, r_{+}^{\prime}\right)+b_{1}^{+}\left(a, r_{-}^{\prime}\right)\right)
$$

where $b_{1}^{+}$is given by (15) i.e.,

$b^{0}\left(x, x^{\prime}, \xi\right)=\frac{1}{2 \pi^{2} a^{3}} \delta\left(r_{+}-a\right)\left(\alpha_{1}\left(a, r_{+}^{\prime}\right)-\alpha_{1}\left(a, r_{-}^{\prime}\right)+\beta_{1}\left(a, r_{-}^{\prime}\right)\right)+\frac{1}{2 \pi^{2} a^{3}} \delta\left(r_{-}-a\right)\left(\alpha_{1}\left(a, r_{-}^{\prime}\right)-\alpha_{1}\left(a, r_{+}^{\prime}\right)+\beta_{1}\left(a, r_{+}^{\prime}\right)\right)$

where $\alpha_{1}(a, r)$ and $\beta_{1}(a, r)$ are given by (10) and (11).

In the rarefied gas approximation the total bias is additive, i.e.,

$$
b_{\text {total }}\left(x, x^{\prime}\right)=\sum b^{0}\left(x, x^{\prime}, \xi_{i}\right)=\int b^{0}\left(x, x^{\prime}, \xi\right) F(\xi) d \xi
$$

where $F$ is given by

$$
\begin{gathered}
F(\xi, N)=\sum_{A=1}^{N} \delta\left(\xi-\xi_{A}\right) . \\
b^{0}\left(x, x^{\prime}\right)=\int \frac{1}{2 \pi^{2} a^{3}} \delta\left(\left|x-R_{+}\right|-a\right)\left(\alpha_{1}\left(a,\left|x^{\prime}-R_{+}\right|\right)-\alpha_{1}\left(a,\left|x^{\prime}-R_{-}\right|\right)+\beta_{1}\left(a,\left|x^{\prime}-R_{-}\right|\right)\right) F(\xi) d \xi+(+\leftrightarrow-)
\end{gathered}
$$

Fist, we point out the obvious symmetry of $F(\xi)$ in the replacement $(+\leftrightarrow-)$ which means that the two above terms coincide. Then, we transform $R_{ \pm}-x^{\prime} \rightarrow R_{ \pm}$which gives

$$
b^{0}\left(x, x^{\prime}\right)=2 \int \frac{1}{2 \pi^{2} a^{3}} \delta\left(\left|x-x^{\prime}-R_{+}\right|-a\right)\left(\alpha_{1}\left(a,\left|R_{+}\right|\right)-\alpha_{1}\left(a,\left|R_{-}\right|\right)+\beta_{1}\left(a,\left|R_{-}\right|\right)\right) F\left(a, R_{+}+x^{\prime}, R_{-}+x^{\prime}\right) d \xi .
$$

For a homogeneous and isotropic distribution $F(\xi)=F(a, X), X=R_{+}-R_{-}$then for the bias we find

$$
b^{0}\left(x-x^{\prime}\right)=2 \int \frac{\alpha_{1}(a)}{2 \pi^{2} a^{3}} \delta\left(\left|x-x^{\prime}-R_{+}\right|-a\right)\left(\frac{K_{1}\left(m R_{+}\right)}{m R_{+}}-\left(1-\frac{\beta_{1}(a)}{\alpha_{1}(a)}\right) \frac{K_{1}\left(m R_{-}\right)}{m R_{-}}\right) F(a, X) d \xi
$$

where $\alpha_{1}(a) \frac{K_{1}\left(m R_{+}\right)}{m R_{+}}=\alpha_{1}\left(a,\left|R_{+}\right|\right)$, etc.. For the Fourier transform $b(k)=\int b(x) e^{i k x} d^{4} x$ and $F(a, k)=$ $\int F(a, X) e^{i k x} d^{4} x$ we get

$$
b_{\text {total }}(k)=-\frac{4 \pi^{2}}{k^{2}+m^{2}} \nu(k)
$$


where

$$
\nu(k)=-\int \frac{2 \alpha_{1}(a)}{m^{2}}\left(F(a, 0)-\left(1-\frac{\beta_{1}(a)}{\alpha_{1}(a)}\right) F(a, k)\right) \frac{J_{1}(k a)}{k a / 2} d a,
$$

and we used the obvious properties $m^{2} \frac{K_{1}(m x)}{m x}=\int \frac{4 \pi^{2}}{k^{2}+m^{2}} e^{-i k x} \frac{d^{4} k}{(2 \pi)^{4}}$ and $\int e^{i k y} \delta(|y|-a) d^{4} y=2 \pi^{2} a^{3} \frac{J_{1}(k a)}{k a / 2}$.

The bias (29) is defined in the linear approximation only. Now by the use of iterations (20), (21) we find $N(k)=$ $1+b_{\text {total }}(k)+b_{\text {total }}^{2}(k)+\ldots$ and true Green function in a gas of wormholes becomes

$$
G_{\text {true }}=G_{0}(k) N(k)=\frac{4 \pi^{2}}{k^{2}+m^{2}+4 \pi^{2} \nu(k)} .
$$

At sufficiently large scales $2 \pi / k \gg \ell_{p l} \sim a,\left|R_{+}-R_{-}\right|$we can expand the function $\nu(k)$ as $\nu(k) \approx \nu(0)+\frac{1}{2} \nu^{\prime \prime}(0) k^{2}$ which defines the renormalized Green function as

$$
G_{r e n}(k)=\frac{4 \pi^{2} Z}{k^{2}+M^{2}}
$$

where

$$
Z=1 /\left(1+\frac{1}{2} \nu^{\prime \prime}(0)\right)
$$

defines the renormalization of charge values due to the polarization of the gas of virtual wormholes and

$$
M^{2}=\left(m^{2}+4 \pi^{2} \nu(0)\right) / Z
$$

that of the mass. We point out that in general $\nu(0)$ possesses both signs depending on the sign of $\zeta$. At very small scales $k \rightarrow \infty$ the function $\nu(k)$ defines the spectral density of states of the scalar field [11] as $N(k)=$ $\left(k^{2}+m^{2}\right) /\left(k^{2}+m^{2}+4 \pi^{2} \nu(k)\right)$.

The spacetime foam picture assumes that typical virtual wormholes have throats $a \lesssim \ell_{p l} \sim 10^{-33} \mathrm{~cm}$ and therefore we can restrict to the case when $m a \ll 1$. Then in the limit $m a \rightarrow 0$ we find [17] $\alpha_{1}(a)=-\frac{1-6 \zeta}{2(1-3 \zeta)} m^{2} a^{2}, \beta_{1}(a)=$ $\frac{6 \zeta}{2(1-3 \zeta)} m^{2} a^{2}$ and the function $\nu(k)$ becomes

$$
\nu(k)=-\int a^{2} \frac{F(a, k)-(1-6 \zeta) F(a, 0)}{(1-3 \zeta)} \frac{J_{1}(k a)}{k a / 2} d a .
$$

The non-minimal coupling $\zeta \neq 0$ generates an additional source at wormhole throats, which in the case $m=0$ brakes the Gauss theorem $\left(\oint \frac{\partial G}{\partial n} d S=-4 \pi^{2}\right)$. Therefore, it is convenient to split the bias into two parts $\nu(k, \zeta)=$ $\nu(k, 0)+\mu(k, \zeta)$ where the first part is

$$
\nu(k, 0)=\int a^{2}(F(a, 0)-F(a, k)) \frac{J_{1}(k a)}{k a / 2} d a
$$

and the second part is

$$
\mu(k, \zeta)=-\frac{3 \zeta}{(1-3 \zeta)} \int a^{2}[(F(a, k)+F(a, 0))] \frac{J_{1}(k a)}{k a / 2} d a .
$$

In the long wave limit $k \rightarrow 0$ one gets $F(a, k) \approx F(a, 0)+\frac{1}{2} F^{\prime \prime}(a, 0) k^{2}$ (for the isotropic distribution of wormholes $F^{\prime}(a, 0)=0$ and $\left.F^{\prime \prime}(a, 0)<0\right)$ and one gets

$$
\nu(k, 0) \approx k^{2} \int a^{2} \frac{\left(-F^{\prime \prime}(a, 0)\right)}{2} d a
$$

i.e., $\nu(k, 0) \rightarrow 0$, as $k \rightarrow 0$ which in the massless $(\zeta=0)$ case defines a partial screening of the source (e.g., see [11]) $N(0) \rightarrow 1 /\left(1+2 \pi^{2} \int a^{2}\left(-F^{\prime \prime}(a, 0)\right) d a\right)$. The second part may describe the so-called dark source

$$
\mu(0, \zeta)=-\frac{6 \zeta}{(1-3 \zeta)} \int a^{2} F(a, 0) d a
$$

whose sign depends on the value of $\zeta$ and which generates or merely renormalizes the mass (32). 


\section{SUMMARY}

Consider first an example of a finite density of wormholes.

In the case of a finite density of wormholes the space remains to be Euclidean at very small scales and therefore $N(k) \rightarrow 1$ as $k \rightarrow \infty$. To illustrate this we consider now a particular form for $F(a, X)$, e.g.,

$$
F(a, X)=\frac{n}{2 \pi^{2} r_{0}^{3}} \delta\left(a-a_{0}\right) \delta\left(X-r_{0}\right),
$$

where $n=N / V$ is the density of wormholes. This function corresponds to a set of wormholes with the fixed throat size $a_{0}$ and the distance between throats $r_{0}=\left|R_{+}-R_{-}\right|$. Then $F(a, k)=\int F(a, X) e^{i k x} d^{4} x$ reduces to $F(a, k)=n \frac{J_{1}\left(k r_{0}\right)}{k r_{0} / 2} \delta\left(a-a_{0}\right)$. Thus from (29) we find

$$
\nu(k)=-2 n \frac{\alpha_{1}\left(a_{0}\right)}{m^{2}}\left(1-\left(1-\frac{\beta_{1}\left(a_{0}\right)}{\alpha_{1}\left(a_{0}\right)}\right) \frac{J_{1}\left(k r_{0}\right)}{k r_{0} / 2}\right) \frac{J_{1}\left(k a_{0}\right)}{k a_{0} / 2} .
$$

In the limit $m a \ll 1$ we get (compare with the analogous expression in [1] )

$$
\begin{gathered}
\nu(k, 0)=n a_{0}^{2}\left(1-\frac{J_{1}\left(k r_{0}\right)}{k r_{0} / 2}\right) \frac{J_{1}\left(k a_{0}\right)}{k a_{0} / 2} \\
\mu(k, \zeta)=-\frac{3 \zeta}{(1-3 \zeta)} n a_{0}^{2}\left(1+\frac{J_{1}\left(k r_{0}\right)}{k r_{0} / 2}\right) \frac{J_{1}\left(k a_{0}\right)}{k a_{0} / 2}
\end{gathered}
$$

As $k \rightarrow 0$ we get $J_{1}\left(k r_{0}\right) / \frac{k r_{0}}{2} \approx 1-\frac{1}{2}\left(\frac{k r_{0}}{2}\right)^{2}+\ldots$ which gives

$$
\nu(k) \approx \frac{n a^{2} r_{0}^{2}}{8(1-3 \zeta)} k^{2}-\frac{6 \zeta n a^{2}}{1-3 \zeta} .
$$

Which defines $\left.Z=1 /\left(1+\frac{n a^{2} r_{0}^{2}}{8(1-3 \zeta)}\right)\right)$ and $M^{2}=\left(m^{2}-4 \pi^{2} n a^{2} \frac{6 \zeta}{1-3 \zeta}\right) / Z$. Thus, we see that in the long-wave limit $\left(k a, k r_{0} \ll 1\right)$ the presence of a particular set of virtual wormholes renormalizes merely the value of the charge and mass values. In the short-wave limit $k \rightarrow \infty$ we get $\frac{J_{1}(z)}{z / 2} \approx \sqrt{\frac{8}{\pi z^{3}}} \cos (z-3 \pi / 4)+\ldots$ and $\nu(k) \rightarrow 0$ as

$$
\nu(k) \approx \frac{n a^{2}}{(1-3 \zeta)}\left(1-6 \zeta-\sqrt{\frac{8}{\pi\left(k r_{0}\right)^{3}}} \cos \left(k r_{0}-\frac{3 \pi}{4}\right)\right) \sqrt{\frac{8}{\pi\left(k a_{0}\right)^{3}}} \cos \left(k a_{0}-\frac{3 \pi}{4}\right),
$$

i.e., the bare parameters of the field theory restore $Z \rightarrow 1$ and $M \rightarrow m$ which means that in the presence of a finite density of wormholes at sufficiently small scales space looks like the ordinary Euclidean space.

The case of limiting topologies or infinite densities of wormholes

Consider now the limiting distribution when the density of wormholes $n \rightarrow \infty$. Since every throat cuts the finite portion of the volume $\frac{\pi^{2}}{2} a^{4}$, this case requires considering the limit $a \rightarrow 0$, i.e., wormhole throats degenerate into points and the minimal scale below which the space looks like the Euclidean space is merely absent. We assume that in this limit $a^{2} F(a, X)=\delta(a) f(X)$ where $f(X)$ is a finite specific distribution. Then (33) reduces to

$$
\nu(k)=(\widetilde{f}(0)-\widetilde{f}(k))-\frac{3 \zeta}{(1-3 \zeta)}(\widetilde{f}(k)+\widetilde{f}(0))
$$

where $\widetilde{f}(k)=\int f(X) e^{i k X} d^{4} X$ (in particular, for conformal coupling $\zeta=\frac{1}{6}$ we get simply $\nu(k)=-2 \widetilde{f}(k)$ ). Now if we require that $\tilde{f}(k)$ is chosen in such a way that

$$
G_{\text {true }}\left(x-x^{\prime}=0\right)=\int \frac{4 \pi^{2}}{k^{2}+m^{2}+4 \pi^{2} \nu(k)} \frac{d^{4} k}{(2 \pi)^{4}}<\infty,
$$

then quantum field theory in such spaces turns out to be finite. We recall that upon continuation to the Minkowsky space the Euclidean Green function transforms to the Feynman propagator $G\left(x-x^{\prime}\right) \rightarrow 4 \pi^{2} D_{F}\left(x-x^{\prime}\right)$. In other words we get here a class of limiting topologies where Green functions (31) $G_{\text {true }}$ or the Feynman propagator $D_{F}$ have a good ultraviolet behavior and QFT is free of divergencies, e.g., see [14, 15]. We also point out that the explicit form of $\nu(k)$ can be found numerically in the lattice quantum gravity e.g., see [2 [4] and references therein. 


\section{ACKNOWLEDGMENT}

I would like to acknowledge A. Kirillov for valuable discussions and comments.

[1] Wheeler J.A., 1964 in: Relativity, Groups, and Topology, B.S. and C.M. DeWitt (eds. ), Gordan and Breach, New York; S.W. Hawking, Nuclear Phys., B114 (1978) 349.

[2] J. Ambjorn, J. Jurkiewicz, and R. Loll, Phys. Rev. Lett. 95, 171301 (2005)

[3] E. Manrique, S. Rechenberger and F. Saueressig, Asymptotically Safe Lorentzian Gravity. Phys. Rev. Lett. 106 (2011) 251302, 1-4

[4] J. Laiho and D. Coumbe, Evidence for Asymptotic Safety from Lattice Quantum Gravity. Phys. Rev. Lett. 107 (2011), 161301

[5] A.A. Kirillov, E.P. Savelova, Phys. Lett. B 660 (2008) 93; Gravitation and Cosmology, (2008) 14, 256-261

[6] Hawking, S.W., Phys Rev. D, 1988, vol. 37, p. 904

[7] Lavrelashvili, G.V., Rubakov, V.A., and Tinyakov, P.G., Nucl. Phys. B, 1988, vol. 299, p. 757

[8] Strominger, A., Phys. Rev. Lett., 1984, vol. 52, p. 1733; Horowitz, G.T., Class. Quant. Grav., 1991, vol. 8, p. 587; Carlip, S., Class. Quant. Grav., 1998, vol. 15, p. 2629; Borde, A., Dowker, H.F., Garcia, R.S., Sorkin, R.D., and Surya, S., Class. Quant. Grav., 1999, vol. 16, pp. 3457-3481.

[9] Biller, S.D., Breslin, A.C., et. al., Phys. Rev. Lett., 1999, vol. 83, p. 2108; Fogli, G.L., Lisi, E., Marrone, A., and Scioscia, G., Phys. Rev. D, 1999, vol. 60, 053006 (hep-ph/9904248); Lisi, T., Marrone, A., and Montanino, D., Phys. Rev. Lett., 2000, vol. 85, p. 1166 (hep-ph/0002053); Ellis, J., Lopes, J., Mavromatos, N., and Nanopoulos, D., Phys. Rev. D., 1996, vol. 53, p. 3846; Benatti, F. and Floreanini, R., Phys. Lett. B, 1997, vol. 401, p. 337; Benatti, F. and Floreanini, R., Phys. Lett. B, 1999, pp. 451, 422; Domokos, G. and Kovesi-Domokos, S., J. Phys. A, 1999, vol. 32, p. 4105

[10] A.A. Kirillov, E.P. Savelova, Artificial Wormhole, arXive:1204.0351

[11] A.A. Kirillov, E.P. Savelova, Dark energy from the gas of wormholes, arXive:1006.5181

[12] V.A. Fock, Z. Phys., 98 (1935) 148

[13] Taub, A. H. (1980). Space-times with distribution valued curvature tensors, Journal of Mathematical Physics 21, 14231431

[14] J. Martin, arXive: 1205.3365v1

[15] T.-P. Cheng, L.F. Li, Gauge theory of elementary particles, Clarendon Press, Oxford, 1984

[16] Loss of coherence must lead to damping of oscillations

[17] In the opposite case $m a \rightarrow \infty$ (macroscopic virtual wormholes) we get $\alpha_{1}\left(a, r^{\prime}\right)=-(1-12 \zeta) \frac{1}{\sqrt{2 \pi m a}} e^{m a}$, and $\beta_{1}\left(a, r^{\prime}\right)=$ $\sqrt{\frac{2 m a}{\pi}} e^{m a}$ 